\title{
DEPSO: Hybrid Particle Swarm with Differential Evolution Operator
}

\author{
Wen-Jun Zhang, Xiao-Feng Xie* \\ Institute of Microelectronics, \\ Tsinghua University, \\ Beijing 100084, P. R. China \\ *Email: xiexiaofeng@ tsinghua.org.cn
}

\begin{abstract}
A hybrid particle swarm with differential evolution operator, termed DEPSO, which provide the bell-shaped mutations with consensus on the population diversity along with the evolution, while keeps the selforganized particle swarm dynamics, is proposed. Then it is applied to a set of benchmark functions, and the experimental results illustrate its efficiency.
\end{abstract}

Keywords: Particle swarm optimization, differential evolution, numerical optimization.

\section{Introduction}

Particle swarm optimization (PSO) is a novel multiagent optimization system (MAOS) inspired by social behavior metaphor [12]. Each agent, call particle, flies in a D-dimensional space $S$ according to the historical experiences of its own and its colleagues. The velocity and location for the $i$ th particle is represented as $\vec{v}_{i}=$ $\left(v_{i 1}, \ldots, v_{i d}, \ldots, v_{i \mathrm{D}}\right)$ and $\vec{x}_{i}=\left(x_{i 1}, \ldots, x_{i d}, \ldots, x_{i \mathrm{D}}\right)$, respectively Its best previous position is recorded and represented as $\vec{p}_{i}=\left(p_{i 1}, \ldots, p_{i d}, \ldots, p_{i \mathrm{D}}\right)$, which is also called pbest. The index of the best pbest is represented by the symbol $g$, and $\vec{p}_{g}$ is called gbest. At each step, the particles are manipulated according to the following equations [15]:

$$
\begin{array}{cc}
v_{i d}=w \cdot v_{i d}+c_{1} \cdot \operatorname{rand}() \cdot\left(p_{i d}-x_{i d}\right)+c_{2} \cdot \operatorname{rand}() \cdot\left(p_{g d}-x_{i d}\right) & (1 \mathrm{a}) \\
x_{i d}=x_{i d}+v_{i d}
\end{array}
$$

where $w$ is inertia weight, $c_{1}$ and $c_{2}$ are acceleration constants, rand () are random values between 0 and 1 .

Several researchers have analyzed it empirically [1, $11,20]$ and theoretically $[3,5]$, which have shown that the particles oscillate in different sinusoidal waves and converging quickly, sometimes prematurely, especially for PSO with small $w$ [20] or constriction coefficient[3].

The concept of a more-or-less permanent social topology is fundamental to PSO [10, 12], which means the pbest and gbest should not be too closed to make some particles inactively $[8,19,20]$ in certain stage of evolution. The analysis can be restricted to a single dimension without loss of generality. From equations (1), $v_{i d}$ can become small value, but if the $p_{i d}-x_{i d} \mid$ and $\left|p_{g d^{-}} x_{i d}\right|$ are both small, it cannot back to large value and lost exploration capability in some generations. Such case can be occured even at the early stage for the particle to be the gbest, which the $p_{i d^{-}} x_{i d} \mid$ and $\left|p_{g d^{-}} x_{i d}\right|$ are zero, and $v_{i d}$ will be damped quickly with the ratio $w$. Of course, the lost of diversity for $\left|p_{i d}-p_{g d}\right|$ is typically occured in the latter stage of evolution process.

To maintain the diversity, the DPSO version [20] introduces random mutations on the $x_{i d}$ of particles with small probability $c_{l}$, which is hard to be determined along with the evolution, at least not be too large to avoid disorganization of the swarm. It can be improved by a bell-shaped mutation, such as Gaussian distribution [8], but a function of consensus on the step-size along with the search process is preferable [11]. A bare bones version [11] for satisfying such requirements is to replace the equations (1) by a Gaussian mutation with the mean $\left(p_{i d}+p_{g d}\right) / 2$ and the standard deviation $\left|p_{i d}-p_{g d}\right|$, which maybe also be inefficient when $\left|p_{i d}-p_{g d}\right|$ is very small, and is too radically since it turns the PSO into a variaty of in evolution strategies (ES) [2]. 
This paper describes a hybrid particle swarm with differential evolution (DE) operator[16], termed DEPSO, which also provide the bell-shaped mutations with consensus on the population diversity, while keeps the particle swarm dynamics. Then the DEPSO is applied to several benchmark functions [4, 13, 15], and the results illustrate the significant performance improvement.

\section{DEPSO algorithm}

For proposed DEPSO, the mutations are provided by DE operator [16] on the $\vec{p}_{i}$, with a trail point $\vec{T}_{i}=\vec{p}_{i}$, which for $d$ th dimension:

$$
\text { IF }(\operatorname{rand}()<C R \text { OR } d==k) \text { THEN } T_{i d}=p_{g d}+\delta_{2, d}
$$

where $k$ is a random integer value within [1, D], which ensures the mutation at least one dimension., $C R$ is a crossover constant, and $\vec{\delta}_{2}$ is the case of $N=2$ for the general difference vector $\vec{\delta}_{N}$, which is defined as:

$$
\vec{\delta}_{N}=\left(\sum_{1}^{N} \vec{\Delta}\right) / N
$$

where the $\vec{\Delta}$ is the essential difference vector [16], means the diffe rence of two elements that random chosen from a common point set, which include all the pbest in current case. $N$ is the number of $\vec{\Delta}$ involved. Then for the $d$ th dimension:

$$
\vec{\Delta}_{d}=\vec{p}_{A, d}-\vec{p}_{B, d}
$$

where $\vec{p}_{A}, \vec{p}_{B}$ are chosen from the pbest set at random.

The experimental analysis f or $\vec{\delta}_{N}$ will be restricted to a single dimension without loss of generality. Fig. 1 and 2 shows a histogram of points that were tested in one million iterations for $\vec{\delta}_{1}$ and $\vec{\delta}_{2}$, respectively. Each one dimensional element $\vec{p}$ for calculating $\vec{\Delta}$ is a realvalue that picked from $[-1,1]$ at random. It can be seen that $\vec{\delta}_{1}$ is a triangle distribution and $\vec{\delta}_{2}$ is a bell-shaped distribution, which the latter is better for problemsolving [11]. Hence the $N=2$ is selected in equation (2).

The mutation is performed on the pbest instead of $\vec{x}_{i}$ $[8,20]$ so as to prevent the swarm from disorganizing by unexpected fluctuations, since the replacement of pbest will follow the steady-state strategy, i.e., $\vec{T}_{i}$ will replace $\vec{p}_{i}$ only if it is better than $\vec{p}_{i}$.

The mutation is also based on $\vec{p}_{g}$ provides the social learning capability, which might speed up convergence.
The learning ratio is determined by $C R$, which is the counterpart of interaction probability IP [11]. If $C R=1$, then the equation (2) becomes a bell-shaped mutation operator on $\vec{p}_{g}$ as ES [2]. If $C R<1$, it may retain some dimensions of $\vec{p}_{i}$, which will facilitate the convergence for the fitness landscape that some dimensions are irrelevantly. Such capability is also implemented implicitly in the some former mutation versions $[8,20]$ but is not in the canonical version.

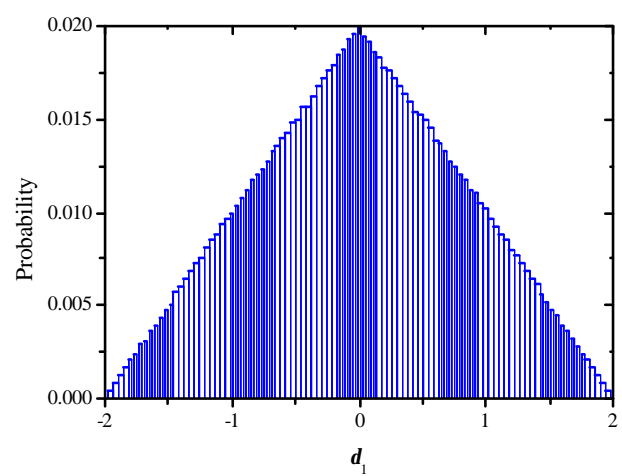

FIG. 1 Histogram of points tested for $\vec{\delta}_{1}$

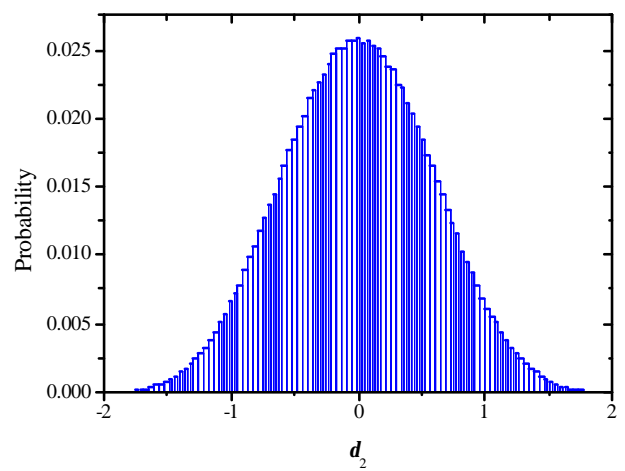

FIG. 2 Histogram of points tested for $\vec{\delta}_{2}$

The original PSO operator and the DE operator will be performed alternately, i.e. the equations (1) will be performed at the odd generations, and the equation (2) at the even generations. The $\vec{\delta}_{2}$ will provide a consensus mutation on $\vec{p}_{i}$ along with diversity of swarm, which emerge from the nature of the search itself, while try ing to keep the diversity of pbest and gbest by changing $\vec{p}_{i}$.

\section{Results and discussions}

For DEPSO, $c_{1}=c_{2}=2, w=0.4$, the maximum velocity $V_{M A X}$ was set to the half range of the search space on each dimension $[9,20]$. If without declaration specially, 
each test case ran for $T=2000$ generations and all the cases were run 100 runs each.

The constraint-handling method is following the criteria [6]: a) any feasible solution is preferred to any infeasible solution; b) among two feasible solutions, the one having better objective function value is preferred; c) among two infeasible solutions, the one having smaller constraint violation is preferred.

The boundary constraints are handled by Periodic mode. For each point $\vec{x}_{i}$, its fitness will be calculated by a mapping point $\vec{z}_{i}=\left(z_{i 1}, \ldots, z_{i d}, \ldots, z_{i \mathrm{D}}\right)$, which for $d$ th dimension, it has:

$$
\begin{aligned}
& \text { IF } x_{i d}<l_{d} \text { THEN } z_{i d}=u_{d}-\left(l_{d}-x_{i d}\right) \% s_{d} \\
& \text { IF } x_{i d}>u_{d} \text { THEN } z_{i d}=l_{d}+\left(x_{i d^{-}} u_{d}\right) \% s_{d}
\end{aligned}
$$

Where ' $\%$ ' is the modulus operator, $l_{d}$ and $u_{d}$ are lower and upper values, and $s_{d}=\left|u_{d}-l_{d}\right|$ is the parameter range of the $d$ th dimension.

\subsection{Unconstrained functions}

The tested unconstrained problems include the Rosenbrock function, the generalized Rastrigrin and the generalized Griewank function $[15,19,20]$, which all in $30-\mathrm{D}$ and have same minimumvalue $\left(F_{\text {opt }}\right)$ as zero.

Table 1 lists the mean fitness values of such functions by FPSO [15], DPSO ( $\left.w=0.4, c_{l}=0.001\right)$ [20], and DEPSO ( $w=0.4, C R=0)$ with different number of agents $(m)$. The $C R$ is set small, since there is little correlation between the parameters for these functions. It shows that DEPSO performs better than both old PSO versions, especially for the Rastrigrin and the Griewank functions, which with uncorrelated parameters.

TABLE 1. The mean fitness values for the unconstrained functions

\begin{tabular}{|c|c|r|r|r|}
\hline$f$ & $m$ & FPSO[15] & DPSO $[20]$ & \multicolumn{1}{c|}{ DEPSO } \\
\hline 30-D \\
Rosenbrock & 20 & 183.8037 & 132.1512 & 80.8259 \\
\cline { 2 - 5 }$\left(F_{\text {opt }}=0\right)$ & 40 & 175.0093 & 82.7209 & 66.8730 \\
\cline { 2 - 5 } & 80 & 124.4184 & 57.2802 & 60.6405 \\
\hline $\begin{array}{c}30-\mathrm{D} \\
\text { Rastrigrin } \\
\left(F_{\text {opt }}=0\right)\end{array}$ & 20 & 48.47555 & 7.3258 & 0.8656 \\
\cline { 2 - 5 } & 40 & 35.20146 & 6.2107 & 0.009950 \\
\hline $\begin{array}{c}30-\mathrm{D} \\
\text { Griewank } \\
\left(F_{\text {opt }}=0\right)\end{array}$ & 20 & 22.52393 & 4.2265 & $3.919 \mathrm{E}-9$ \\
\cline { 2 - 5 } & 40 & 0.021560 & 0.01793 & 0.009073 \\
\cline { 2 - 5 } & 80 & 0.014945 & 0.01360 & 0.006930 \\
\hline
\end{tabular}

\subsection{Constrained functions}

The selected problems include eleven functions that are proposed by Z. Michalewicz et al $\left(G_{1}\right.$ to $\left.G_{11}\right)$ [13], which include eight functions without equality constraints and three functions (1.e. $G_{3}, G_{5}, G_{11}$ ) with equality constraints, and an engineering optimization problem: design of a pressure vessel (Vessel) [4, 9]. The dimension of $S$ (D), the optimization type and optimum value $\left(F_{\text {opt }}\right)$ of each function are list in Table 2.

T ABLE 2. Summary of constrained functions

\begin{tabular}{|c|r|c|r|}
\hline$f$ & \multicolumn{1}{|c|}{$\mathrm{D}$} & Type & \multicolumn{1}{|c|}{$F_{\text {opt }}$} \\
\hline$G_{1}$ & 13 & Minimum & -15 \\
\hline$G_{2}$ & 20 & Maximum & 0.803612 \\
\hline$G_{4}$ & 5 & Minimum & -30665.539 \\
\hline$G_{6}$ & 2 & Minimum & -6961.814 \\
\hline$G_{7}$ & 10 & Minimum & 24.306 \\
\hline$G_{8}$ & 2 & Maximum & 0.095825 \\
\hline$G_{9}$ & 7 & Minimum & 680.630 \\
\hline$G_{10}$ & 8 & Minimum & 7049.248 \\
\hline$G_{3}$ & 10 & Minimum & -1 \\
\hline$G_{5}$ & 4 & Minimum & 5126.498 \\
\hline$G_{11}$ & 2 & Minimum & 0.75 \\
\hline Vessel & 4 & Minimum & 6059.714 \\
\hline
\end{tabular}

Table 3 lists the mean fitness values for eight functions without equality constraints by a $(30,200)$-ES $(T=1750)$ [17], the DE $(C R=0.1)$, the canonical PSO $(w=0.4)$, and the DEPSO $(w=0.4, C R=0.1)$, where $m=70$. For $G_{8}$, the maximum generation $T=200$, and for all the other cases, $T=2000$. It shows that the DEPSO outperforms either the DE or the PSO. By the way, it also provides better results than GA [13] and ES [17] with much less evaluation times.

TABLE 3. The $F_{\text {opt }}$ for the functions without equality constraints

\begin{tabular}{|c|r|r|r|r|}
\hline$f$ & \multicolumn{1}{|c|}{ ES[17] } & \multicolumn{1}{c|}{ DE } & \multicolumn{1}{c|}{ PSO } & \multicolumn{1}{c|}{ DEPSO } \\
\hline$G_{1}$ & -15.000 & -15.000 & -14.945 & -15.000 \\
\hline$G_{2}$ & 0.7820 & 0.7805 & 0.6891 & 0.7868 \\
\hline$G_{4}$ & -30665.5 & -30650.1 & -30665.5 & -30665.5 \\
\hline$G_{6}$ & -6875.94 & -6961.81 & -6961.81 & -6961.81 \\
\hline$G_{7}$ & 24.374 & 25.064 & 25.286 & 24.586 \\
\hline$G_{8}$ & 0.095825 & 0.095825 & 0.095825 & 0.095825 \\
\hline$G_{9}$ & 680.656 & 681.063 & 680.652 & 680.641 \\
\hline$G_{10}$ & 7559.192 & 7565.4 & 7526.6 & 7267.4 \\
\hline
\end{tabular}

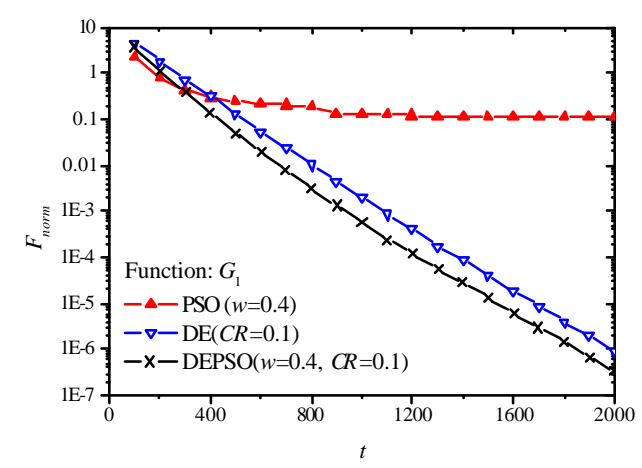

FIG. 3 Mean relative performance for $G_{1}$ 


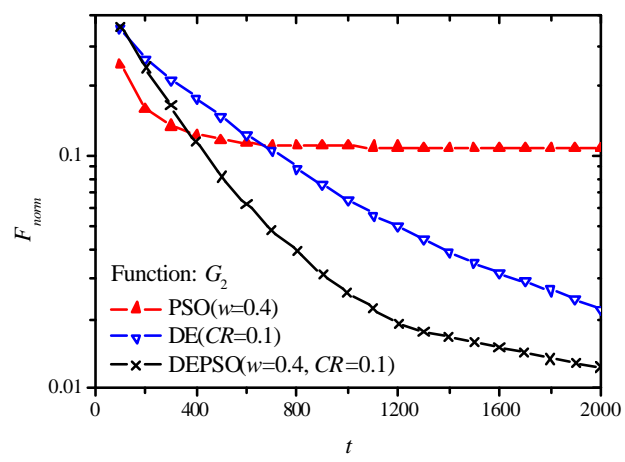

FIG. 4 Mean relative performance for $G_{2}$

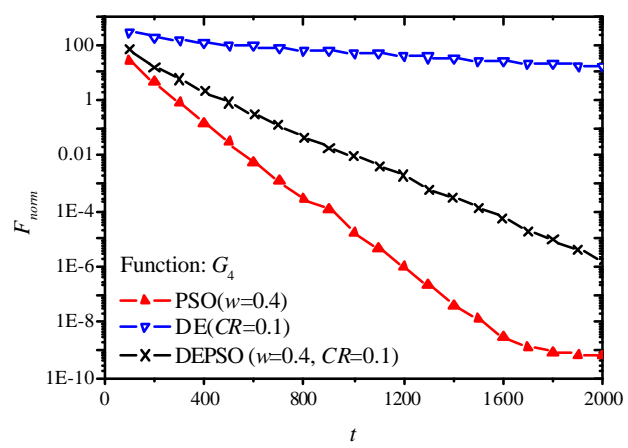

FIG. 5 Mean relative performance for $G_{4}$

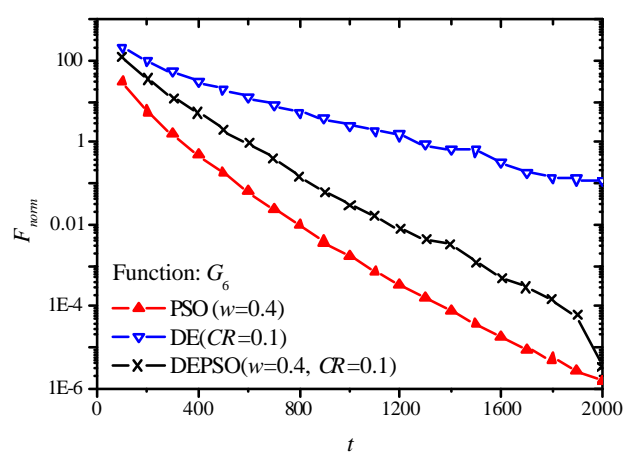

FIG. 6 Mean relative performance for $G_{6}$

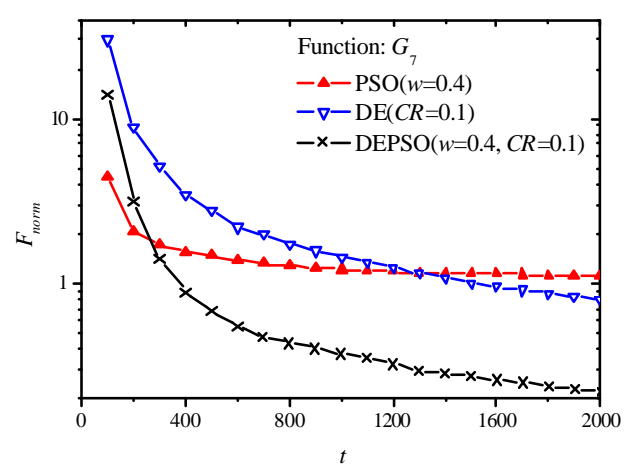

FIG. 7 Mean relative performance for $G_{7}$

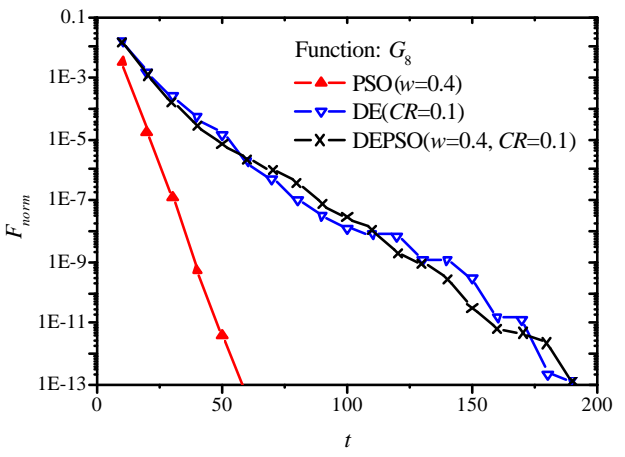

FIG. 8 Mean relative performance for $G_{8}$

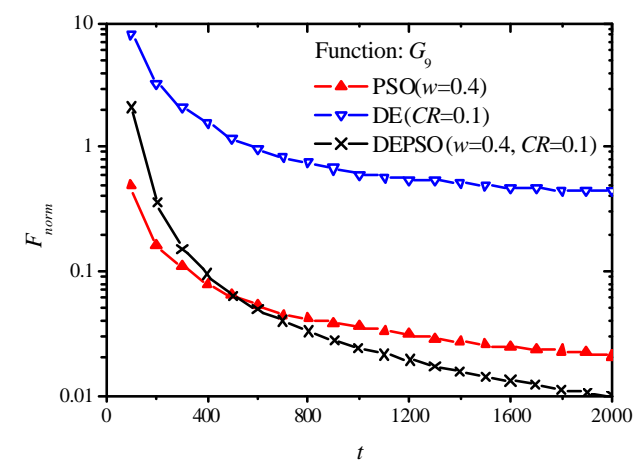

FIG. 9 Mean relative performance for $G_{9}$

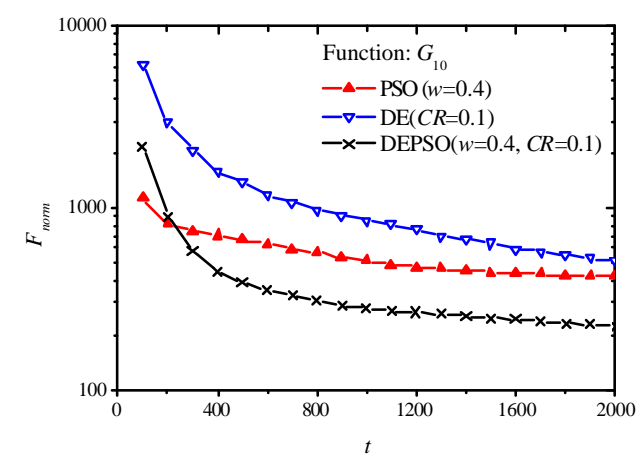

FIG. 10 Mean relative performance for $G_{10}$

Figure 3 to 10 show the relative mean fitness value $F_{\text {norm }}=F_{\text {mean }}-F_{\text {opt }}$ during the evolution generations $(t)$ for the eight functions, respectively, where $F_{\text {mean }}$ is the mean best fitness value in each generation. It shows that DEPSO outperform DE in at least mentioned cases. For some problems, such as $G_{4}, G_{6}, G_{8}$, PSO perform better than DE and DEPSO with $\mathrm{CR}=0.1$, which may due to the fitness landscape of such problems are epistasis, i.e. need to covary the parameters at the same time to improve fitness. Notes although there is no correlation 
between the parameters of $G_{6}$, but its constraints construct such a $S_{F}$. This also can explain why PSO is performing worse for such as $G_{1}$, since lack of the capability of varying only few dimensions for a point.

For $G_{3}, G_{5}, G_{11}$, which have almost $0 \%$ feasible space $\left(S_{F}\right)$ due to the equality constraints, are needed to replace the constraint $g(\vec{x})=0$ by an inequality constraint $|g(\vec{x})|<\varepsilon$ for some small $\varepsilon>0[13,14,17]$. Here we choose two $\varepsilon$ values: 1E-3 [14], and 1E-4 [17], which the $S_{F}$ of the former is larger than that of the latter and will more easily to be solved.

Table 4 lists the mean fitness values for the three functions with equality constraints $\operatorname{DE}(C R=0.9)$, the canonical PSO $(w=0.4)$, and DEPSO $(w=0.4, C R=0.9)$, where $m=70$. In order to compare with existing results, when $\varepsilon=1 \mathrm{E}-3$, for $G_{5}, T=2500$, and for $G_{11}, T=300$; when $\varepsilon=1 \mathrm{E}-4$, for $G_{3}$ and $G_{5}, T=5000$. For other cases, $T=2000$. The values in the brackets gives the number of trails that are failed in entering $S_{F}$, and only those trails that are succeeded in entering $S_{F}$ are counted for the calculation of mean fitness values. DEPSO outperforms DE and PSO in all cases, and it can find the optimum solution in all runs when $\varepsilon=1 \mathrm{E}-3$. Table 5 summarizes the evaluation times for existing DE results in [14] and DEPSO, which shows DEPSO is also much fast.

TABLE 4 . The $F_{\text {opt }}$ for the functions with equality constraints

\begin{tabular}{|c|c|r|r|r|}
\hline$f$ & $\varepsilon$ & \multicolumn{1}{|c|}{ DE } & \multicolumn{1}{c|}{ PSO } & \multicolumn{1}{c|}{ DEPSO } \\
\hline$G_{3}$ & $1 \mathrm{E}-3$ & -0.8572 & -1.0048 & -1.0050 \\
\hline$G_{3}$ & $1 \mathrm{E}-4$ & -0.3985 & -0.8364 & -0.9849 \\
\hline$G_{5}$ & $1 \mathrm{E}-3$ & 5129.50 & $5356.15(10)$ & 5126.484 \\
\hline$G_{5}$ & $1 \mathrm{E}-4$ & 5133.834 & $5334.97(12)$ & 5130.864 \\
\hline$G_{11}$ & $1 \mathrm{E}-3$ & 0.74909 & 0.74941 & 0.74900 \\
\hline$G_{11}$ & $1 \mathrm{E}-4$ & 0.75061 & 0.75459 & 0.74990 \\
\hline
\end{tabular}

TABLE 5. Summary of the evaluation times when $\varepsilon=1 \mathrm{E}-3$

\begin{tabular}{|c|r|r|}
\hline$f$ & DE[14] & DEPSO \\
\hline$G_{3}$ & 8000000 & 140000 \\
\hline$G_{5}$ & 1200000 & 175000 \\
\hline$G_{11}$ & 30000 & 21000 \\
\hline
\end{tabular}

When $\varepsilon=1 \mathrm{E}-4$, DEPSO also cannot always find the optimum point, which is better than normal ES [17] but is worse than ES with stochastic ranking [17] for $G_{3}$ and $G_{5}$ in same evaluation times.

The objective of the vessel problem is to minimize the cost of the material, forming and welding of a cylindrical vessel [4, 9]. It is a mixed-integer-discretecontinuous problem which has four design variables, two are integer and two are continuous. Here the closest integer value will be used to evaluate the fitness although the algorithms still works internally with continuous variables.

TABLE 6. The mean fitness values for the vessel problem

\begin{tabular}{|c|r|r|c|c|}
\hline Results & MGA[4] & DE & PSO & DEPSO \\
\hline Best & 6069.3267 & 6062.740 & 6059.714 & 6059.714 \\
\hline Mean & 6263.7925 & 6126.658 & 6332.784 & 6108.177 \\
\hline Worst & 6403.4500 & 6408.792 & 6820.410 & 6410.087 \\
\hline S.D. & 97.9445 & 76.0619 & 263.4612 & 93.45319 \\
\hline
\end{tabular}

Table 6 lists the comparison of results for the vessel problem by MGA (population size is 50 , and $T=1000$, which costs 50000 evaluation times ) [4], DE $(C R=0.1)$, the canonical PSO $(w=0.4)$, and DEPSO $(w=0.4$, $C R=0.1$, where for the three algorithms, $m=70$, and $T=700$, which costs 49000 evaluation times. It can be seen that the PSO is not stable [9], which may due to the step-type landscape created by integer variables. However, PSO also shows the capability to catch the optimum point comparing to MGA and DE. DEPSO inherits the merits of PSO and DE, and performs much better that MGA within almost same evaluation times.

\section{Conclusions}

This paper presents a hybrid particle swarm with differential evolution operator called DEPSO. The hybrid strategy provides the bell-shaped mutations with consensus on the population diversity by DE operator, while keeps the self-organized particle swarm dynamics, in order to make the performance is not very sensitive to the choice of the strategy parameters as in DE [7]. It is shown to outperform the PSO and DE for a set of benchmark functions. However, more comparative works with different parameter settings for more problems should be performed to provide a full view.

The DEPSO seems performing well for the problems with integer variables by the help of the bell-shaped mutations. However, as declared for PSO [9], it is als o not very efficiently for handling those problems with extremely small feasible space, such as the problems 
with equality constraints. Since each agent in DEPSO (and DE, PSO) only refers to few points pbest and gbest), it cannot employs some strategies (such as stochastic ranking [17]), which needs a big population. Future investigation may employ extending memory with a set of points to satisfy such strategies.

Moreover, according to No-Free-Lunch (NFL) theory [18], taken the problem information into account will improve the performance of algorithm. For DEPSO, the appropriate $C R$ can be chosen if the correlation of the parameters is known. But for the black-box problems, it is still a great challenge to learning such parameters during run-time with efficiently strategies.

\section{Acknowledgements}

The work is supported by the National Hi-Tech Research and Development Program of China (863 project) under Contract No. 2002AA1Z1460. The authors also thank M. Clerc for helpful discussions.

\section{References}

[1] Angeline P J. Evolutionary optimization versus particle swarm optimization: philosophy and performance difference. Annual Conf. on Evolutionary Programming, 1998: 601-610 [2] Beyer H-G, Schwefel H-P. Evolution strategies: a comprehensive introduction. Natural Computing, 2002, 1(1): 3-52

[3] Clerc M, Kennedy J. The particle swarm - explosion, stability, and convergence in a multidimensional complex space. IEEE Trans. on Evolutionary Computation, 2002, 6(1): 58-73

[4] Coello C A C. Theoretical and numerical constrainthandling techniques used with evolutionary algorithms: a survey of the state of the art. Computer Methods in Applied Mechanics and Engineering, 2002, 191(11-12): 1245-1287

[5] Cristian T I. The particle swarm optimization algorithm: convergence analysis and parameter selection. Information Processing Letters, 2003, 85(6): 317-325

[6] Deb K. An efficient constraint handling method for genetic algorithms. Computer Methods in Applied Mechanics and Engineering, 2000, 186(2-4): 311-338

[7] Gäemperle R, Mü ller S D, Koumoutsakos P. A parameter study for differential evolution. WSEAS Int. Conf. on Advances in Intelligent Systems, Fuzzy Systems, Evolutionary Computation, 2002: 293-298

[8] Higashi N, Iba H. Particle swarm optimization with Gaussian mutation. IEEE Swarm Intelligence Symposium, 2003: $72-79$

[9] $\mathrm{Hu} \mathrm{X} \mathrm{H}$, Eberhart $\mathrm{R} \mathrm{C}$, Shi $\mathrm{Y} \mathrm{H}$. Engineering optimization with particle swarm. IEEE Swarm Intelligence Symposium, 2003: 53-57

[10] Kennedy J. The particle swarm: social adaptation of knowledge. IEEE Int. Conf. on Evolutionary Computation, 1997: 303-308

[11] Kennedy J. Bare bones particle swarms. IEEE Swarm Intelligence Symposium, 2003: 80-87

[12] Kennedy J, Eberhart R C. Particle swarm optimization. IEEE Int. Conf. on Neural Networks, 1995: 1942-1948

[13] Koziel S, Michalewicz Z. Evolutionary algorithms, homomorphous mappings, and constrained parameter optimization. Evolutionary Computation, 1999, 7(1): 19-44

[14] Lampinen J. A constraint handling approach for the differential evolution algorithm. Congress on Evolutionary Computation, 2002: 1468-1473

[15] Shi Y H, Eberhart R C. Fuzzy adaptive particle swarm optimization. IEEE Int. Conf. on Evolutionary Computation, 2001: 101-106

[16] Storn R, Price K. Differential evolution - a simple and efficient heuristic for global optimization over continuous spaces. J. Global Optimization, 1997, 11: 341-359

[17] Runarsson T P, Yao X. Stochastic ranking for constrained evolutionary optimization. IEEE Trans. on Evolutionary Computation, 2000, 4(3): 284-294

[18] Wolpert D H, Macready W G. No free lunch theorems for optimization. IEEE Trans. on Evolutionary Computation, 1997, 1(1): 67-82

[19] Xie X F, Zhang W J, Yang Z L. Adaptive particle swarm optimization on individual level. Int. Conf. on Signal Processing, 2002: 1215-1218

[20] Xie X F, Zhang W J, Yang Z L. A dissipative particle swarm optimization. Congress on Evolutionary Computation, 2002: $1456-1461$ 